\title{
Using CAPTCHAs to Index Cultural Artifacts
}

\author{
Qiang Zhu Eamonn Keogh \\ Dept. of Computer Science \& Engineering \\ University of California, Riverside \\ Riverside, CA 92521 \\ \{qzhu, eamonn\}@cs.ucr.edu
}

\begin{abstract}
Rock art, human-made markings on stone, is an important cultural artifact and the earliest expression of abstract thinking. While there are tens of millions of photographs of rock art in existence, there have been no large-scale attempts to organize, classify or cluster them. This omission is not due to a lack of interest, but reflects the extraordinary difficultly of extracting useful data from an incredibly heterogeneous and noisy dataset. As we shall show, rock art is likely to resist efforts of automatic extraction from images for a long time. In this work we show that we can use CAPTCHAs, puzzles designed to tell humans and computers apart, to segment and index rock art. Unlike other CAPTCHAs which operate on inherently discrete data and expect discrete responses, our method considers inherently real-valued data and expects real-valued responses. This creates a challenge which we have overcome by using a recently introduced distance measure. We demonstrate our system is capable of acting as a secure CAPTCHA, while producing data that allows for indexing the rock art.
\end{abstract}

Keywords: CAPTCHA, Image Processing, Cultural Artifacts, Rock Art

\section{INTRODUCTION}

Rock art is the archaeological term for human-made markings on stone, including petroglyphs, carvings into stone surfaces and pictographs, paintings on stone. Fig. 1 illustrates some examples of each, which hint at the astonishing variability of rock art in terms of complexity and appearance.
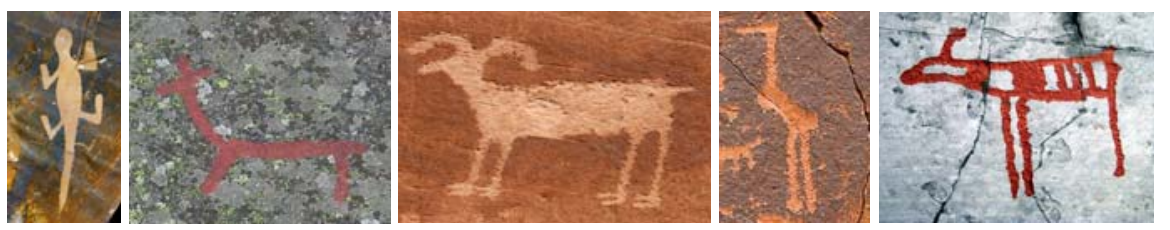

Fig. 1. A selection rock art hints at their incredible variability, complexity and beauty.

Petroglyphs and pictographs are perhaps the earliest expressions of abstract thinking. Studies of rock art have implications beyond anthropology and history. For example, a recent study postulates the existence of a now-extinct Australian bat species based on extraordinarily detailed pictographs known to be at least 17,500 years old [13]. Petroglyphs have been used in studies of climate change; the changing inventories of species in the Dampier Archipelago from the Pleistocene to the early Holocene 
period have been reconstructed partly by petroglyph evidence [4]. However, in spite of these successes, progress in petroglyph research has been frustratingly slow. A decade ago, Walt et al. summed up the state of petroglyph research by noting, "Complete-site and cross-site research thus remains impossible, incomplete, or impressionistic" [15]. Surprisingly, there has been little change in the intervening decade. We believe that this is due only to the difficultly of extracting the petroglyphs from raw images. More concretely, we claim that given the extracted "outline" data, indexing, classification and clustering of rock art would be relatively easy. Since this is the fundamental assumption in our work, let us immediately demonstrate it. In Fig. 2 we have clustered eight rock art images, for which we have (human extracted) "skeleton" data, in the form of a binary bitmap.

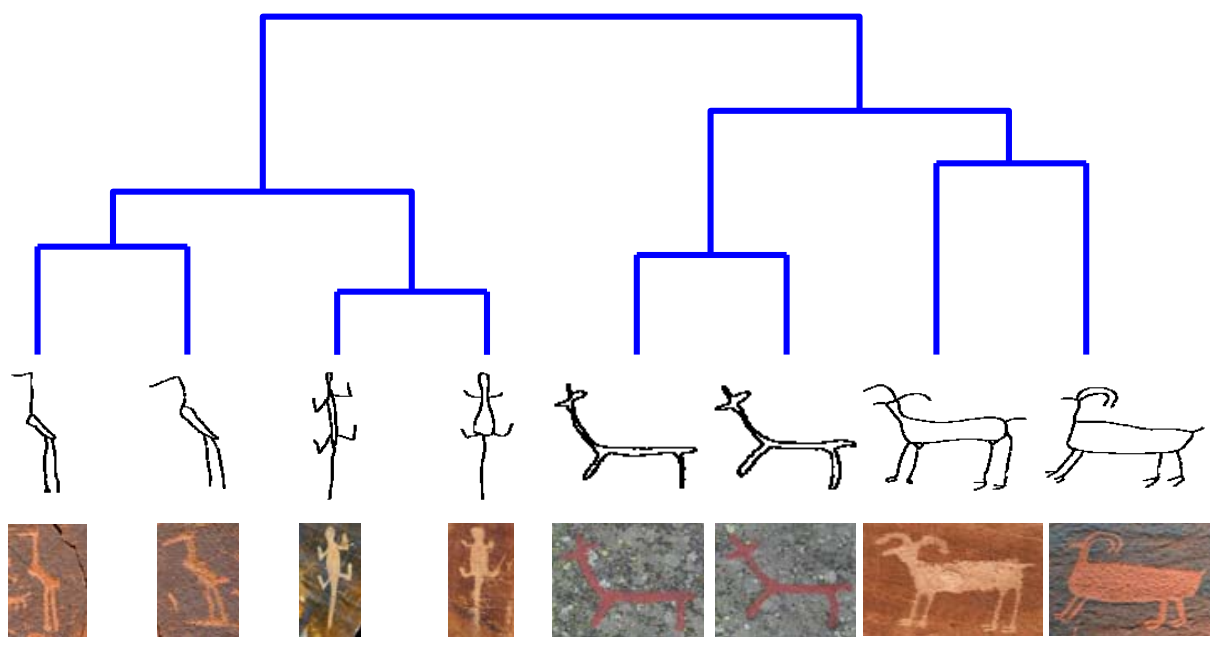

Fig. 2. A clustering of eight petroglyphs using the GHT measure proposed in [16].

This result hints at the fact that if we could only extract the skeletonized data, a wealth of opportunities for anthropological data mining would open up. However, as we shall demonstrate, in the vast majority of cases, the extraction of meaningful data from photographs of rock art is likely to be beyond the capabilities of image segmentation algorithms for a long time. With this in mind, we proposed to extract useful information from unconstrained images of rock art by turning the problem into a CAPTCHA [1]. CAPTCHAs ("Completely Automated Public Turing test to tell Computers and Humans Apart") are tests given by a machine to ensure that a response is generated by a human, not a computer. The most familiar instantiation of them is a sequence of distorted letters that the user must reproduce. Fig. 3 shows two examples.
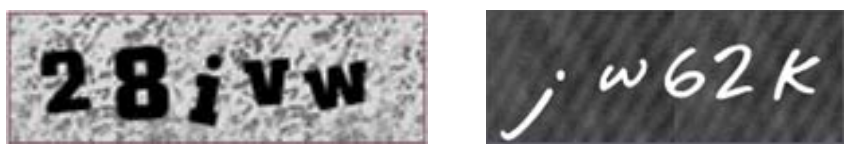

Fig. 3. Two examples of CAPTCHAs. In order to solve the CAPTCHA, and get access to the next webpage (in this case, offering a free email account) the user must type in 28iVW and jw62K respectively. 
These CAPTCHAs operate on inherently discrete data (text, albeit distorted) and expect discrete responses (keystrokes); we can therefore use equality tests to decide if the test was passed, i.e. equals('28ivW','28iVW')?

In contrast, our method considers inherently real-valued data (photographs of rock art) and expects real-valued responses (mouse movements). We cannot expect to test for equality. This creates a significant challenge which we have overcome by using a recently introduced distance measure [16] to test if a tracing of a petroglyph is close enough to a real pattern to indicate human intelligence.

\section{BACKGROUND AND RELATED WORK}

\subsection{Background on Rock Art}

The earliest petroglyphs have traditionally been associated with the appearance of modern humans in Europe such as the famous example from the Lascaux Cave, France, and an early one from the Chauvet Cave, France which dates back to as early as 30,000 years ago [14]. Recent work has shown that the idea of expressing abstract motifs appears much earlier, 77,000 years ago in South Africa [8]. Given this long history, this art is one of the most valuable sources of humanity that has persisted to the present time.

Beyond their value as an aesthetic expression, petroglyphs provide a rich source of information for researchers. Repeated motifs can be identified and traced through time and space, which in turn may shed light on the dynamic histories of human populations, patterns of their migrations and interactions, and even continuities to present indigenous societies. However, the nature of petroglyphs poses an extremely difficult challenge. As in the case of any other artifacts of history, damages to petroglyphs are permanent and irreversible. In addition, unlike other artifacts that can be preserved and protected within the confines of a controlled environment in a museum, petroglyphs are mostly left in their natural settings, exposed to elements of nature that will erode them inevitably with time. There is an urgent need to identify petroglyphs and to archive them for humanity.

An understanding of similarity must be at the heart of any effort to analyze petroglyphs and other cultural artifacts. For example, an image of a horseman incised on a fossilized ostrich eggshell fragment was recently found among eolian deposits in the Gobi Desert, Mongolia [11]. An obvious thing to do with such an image in order to place it in a cultural context is to ask if a similar image exists among the many petroglyphs in the region. In Section 4.2 we show that we can support such queries.

\subsection{Background on Image Processing}

Another fundamental assumption in this work is that there is no automatic segmentation algorithm that can robustly segment rock art. To demonstrate this, we conducted a simple experiment on what is probably one of the most amiable images imaginable, the famous petroglyphs of Alta, Norway. 
We took one image of a reindeer as shown in Fig. 4.left, and tried segmenting it with six different methods: the Sobel method, Prewitt method, Roberts method, Laplacian of Gaussian method, Zero-cross method and Canny method. In each case we spent fifteen minutes adjusting the parameters to achieve the best (subjectively) feature extraction. The best result, using the Prewitt method, is shown in Fig. 4.right.
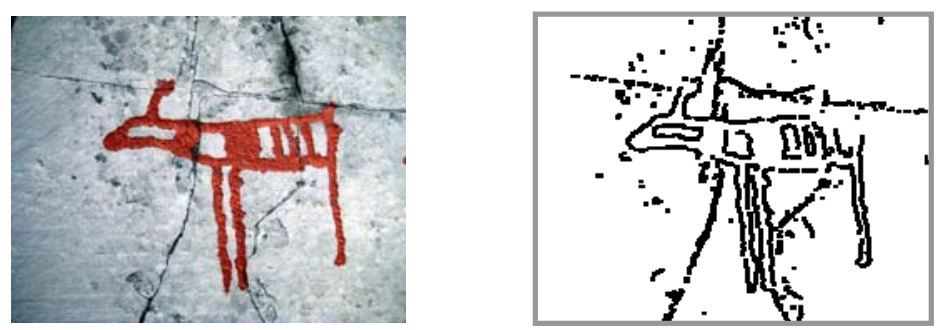

Fig. 4. left) Reindeer rock art from Norway, dating to 4200 to $500 \mathrm{BC}$. The rock carvings have been retouched in bright red by researchers, making them extremely high contrast. right) a segmentation of the image using the Prewitt method, carefully tuned.

Note that while our efforts have paid off in that we have captured much of the animal in question, we are missing a large section of the rump. What is worse, we have many spurious lines corresponding to cracks in the rock. Of course, it is possible that a more sophisticated algorithm could be tuned to do a better job; however, this tuned version is unlikely to generalize to other petroglyphs. Furthermore, it is worth restating that this example is among the highest contrast, cleanest examples of rock art.

\subsection{Background on Human Computation}

The last five years has seen a flurry of research on Human Computation, much of it leveraging off the pioneering work of Luis von Ahn [2]. The essence of human computation is to have computers do as much work as possible to solve a given problem, but to outsource certain critical steps to humans. These steps are ones which are difficult for computers, but simple for humans. One of the most famous examples is the Google Image Labeler, which is a program that allows the user to label random images to help improve the quality of Google's image search results. Like many such efforts, human time is donated for free, because the task is embedded in a fun game; hence the recently coined term, Games with a Purpose, or GWAP [3].

\subsection{Distance Measures for Line Drawings}

As noted in Section 2.1, an effective and robust similarity measure is crucial to mining petroglyphs and, as we shall see, in testing to see if a "skeleton" drawing submitted to a CAPTCHA can be attributed to human intelligence. After soliciting feedback and advice from various researchers in the data mining and image processing community, and testing dozens of possible measures, we proposed a distance measured based on the Generalized Hough Transform (GHT) [16]. The utility of this measure can be subjectively judged in Fig. 2 and objectively measured by classifying hand drawn symbols (Farsi digits, icons, etc.) that are "petroglyph-like" [16]. 
The GHT is a useful method to detect arbitrary two-dimensional shapes [5][12], in which shapes are constituted of edge points (simply the dark pixels in the binary representation of shapes). The goal of GHT is to find the best fit between a query shape $Q$ and a candidate shape $C$. That is, if we place $Q$ onto $C$ (with only translation in the plane allowed), the number of matched edge points should be the maximal.

For clarity and simplicity, here we give a toy example to illustrate how it works. Fig. 5.left shows a query shape $Q$ and candidate shapes $C_{1}$ and $C_{2}$. A best fit between $Q$ and $C_{1}$ is also shown on the right of Fig. 5, and we can consider that $Q$ is found in $C_{1}$ at this particular alignment.
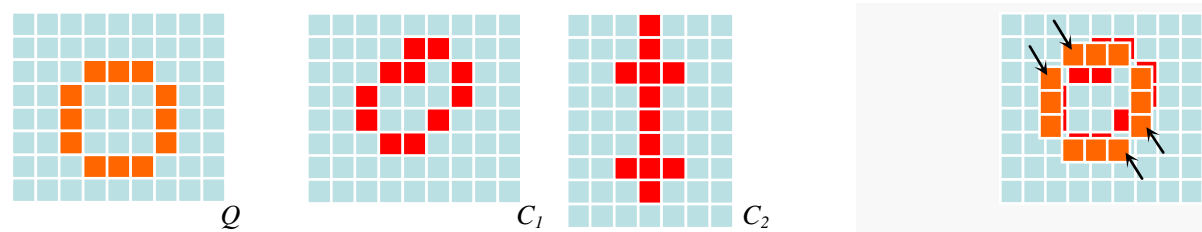

Fig. 5. left) Toy examples of a query $Q$ and candidate matches $C_{1}$ and $C_{2}$. Each cell is a pixel, and the dark colors denote edge points of shapes. right) The GHT aligns $Q$ and $C_{1}$ by maximizing matched edge points between them.

The GHT can effectively detect shapes, but it does not explicitly encode a distance measure. To find a numeric evaluation of it, we defined the minimal unmatched edge points (MUE) of $Q$, which is simply the number of edge points in $Q$ minus maximal matched points. In our example, with the similar shape $C_{1}$, its value is $12-8=4$ (reflected by the four arrows in Fig. 5.right). If we had compared $Q$ to $C_{2}$, the MUE would equal 6 , meaning $Q$ is less similar to $C_{2}$ than $C_{1}$.

We also considered several slight variations of MUE to enable higher-level data mining algorithms, and we will use one of them, the clustering distance variant [16], in all experiments in this paper.

\section{CAPTCHA-ROCK}

\subsection{A Simple Image-based Stickman CAPTCHA}

While the goal of this research is to introduce a method that will allow us to capture data from real photographs of petroglyphs, for ease of exposition we will begin discussing the problem as if our only intention were to produce an image-based CAPTCHA with artificial data.

It is simple to write a program to produce random instances of a "stick figure"; Fig. 6 shows four examples.
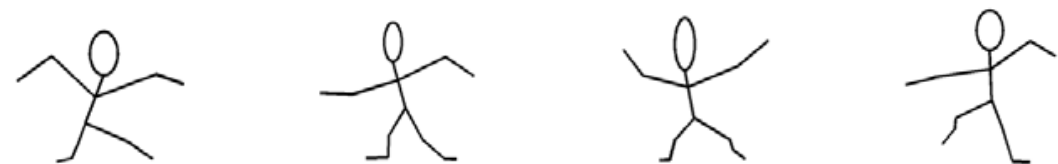

Fig. 6. Four examples of a parameterized Stickman. 
To ensure each stickman is unique (with very high probability), we have parameterized the code. The following features are parameterized:

- The head size and aspect ratio

- The length of the humerus, forearm, femur, tibia and foot

- The angles of knees, elbows, ankle and torso (these may be asymmetric)

There are other elements of a human stick figure that we could represent and parameterize, but this simple model is sufficient for our purposes.

For reasons that we will see shortly, it is useful to ask what the average distance is between two randomly created figures under the GHT-distance measure discussed in the previous section. To calculate this, we generated 1,000 pairs of stickmen and calculated the distance between each pair, summarizing the results in Fig. 7.

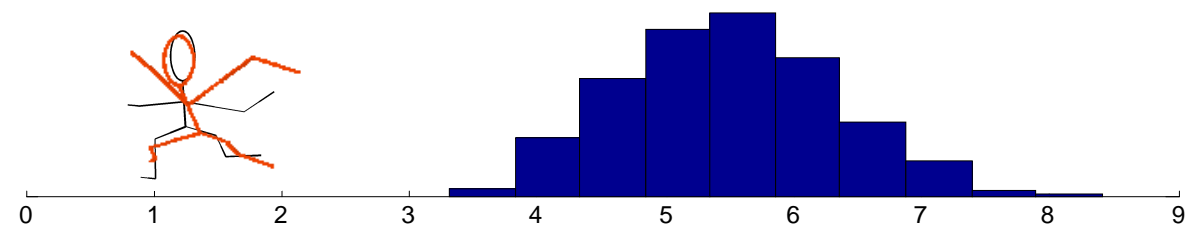

Fig. 7. left) A pair of randomly generated stickmen. right) The distribution of GHT distances between 1,000 pairs of randomly generated stickmen.

If we instead produce random stickmen, and ask humans to trace their outline on the screen with the mouse pointer (as in Fig. 8.left), we might expect the distances between the generated and traced outlines to be generally smaller.

To verify this, we generated 20 stickmen and asked volunteers to trace them. How well a person can trace the stickmen depends on their dexterity, input device, screen size, etc. Given these variations, we asked three volunteers to trace each stickman on their own machines. Fig. 8.right shows the distribution of these distances.

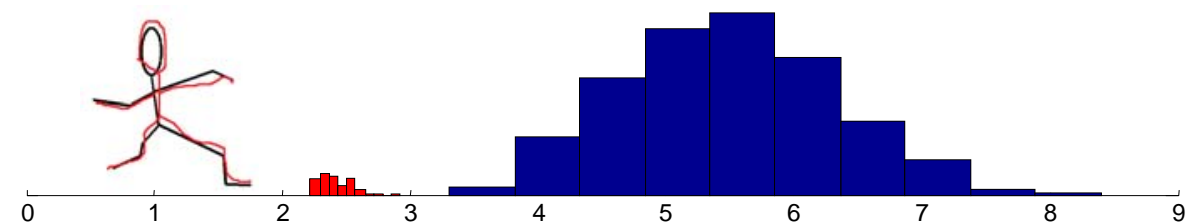

Fig. 8. left) A randomly generated stickman in black and a human tracing of it in red. right) The distribution of GHT distances between randomly generated stickmen and human tracings of them are shown with a finer bucket size (in red), because there is less data. The distribution of GHT distances between two randomly generated stickmen is shown for context (in blue).

It is easy to see that we could use these results to create a simple stickman CAPTCHA. We could produce a stickman, and ask the user to trace it. If a human traces the stickman, we can be near certain that the distance to the template will be less than 3 (from Fig. 8). For simplicity here we assume that the attacker has the code to produce the stickmen, and simply sends a random stickman as his guess. If that is so, his guess will almost certainly be greater than 3 (from Fig. 7/Fig. 8) and we can reject his attempt. Of course the attacker could use an image processing algorithm to produce a "customized guess", and we could counter by imbedding the stickman in field of distracters and distortions, however, a better idea is to find "stickmen" in rock art, the subject of the next section. 


\subsection{A CAPTCHA-ROCK System Helping Extract Data From Petroglyphs}

Motivated by our experiences with the stickmen CAPTCHA, we can now ask: is it possible to design a CAPTCHA system which provides high security (serves as a CAPTCHA) while collecting useful information about rock art (serves as a Human Computation tool)? The reCAPTCHA [2] proposed by Luis von Ahn is the ideal paradigm to follow. In this system, which is designed to transcribe degraded text from scanned books and newspapers, each test gives the user two words to recognize, one of which is a "control word", whose answer is known, and the other one is an unknown word. If the user can correctly type the "control word", the reCAPTCHA assumes that the inputs come from a human and the answer for the "unknown word" is correct (or at least plausible). Once an "unknown word" receives enough "votes" from a same answer, it can become a "control word". This system has already transcribed several hundred million words, which OCR systems failed to parse.

We can use the same idea to build our CAPTCHA-ROCK system. An example is shown in Fig. 9, in which the user is asked to trace both petroglyphs correctly to pass the CAPTCHA.

Please trace both petroglyphs with your mouse pointer
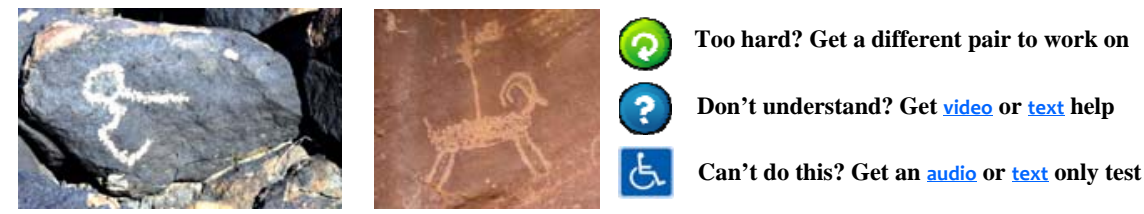

Help with the digitization of important cultural artifacts, visit www.cs.ucr.edu/ eamonn/anthropology/

Fig. 9. A CAPTCHA-ROCK consists of two rock art images: one control image and one unknown image. Note that users do not know which one is which.

There are still three extra problems/questions we must solve to make this work:

- How should we build the initial "control image" set?

To frustrate robots that break challenges by simple random guesses, the set of control words in reCAPTCHA contains more than 100,000 items. Do we also need such a large "control image" set? We believe that the answer is no. We can frustrate an attacker that attempts to simply memorize the entire control image set (with solution tracings) by performing simple scale and translation operations to images. This means that even if the attacker's algorithm correctly detects which image is the control image (perhaps by color), the relative location of the petroglyph within the image may have changed just enough so that even if the attacker sends the right trace, it will be in the wrong location.

As we shall show in the next section, our initial experiments show that even for a control set containing only 143 images, by using these simple scale and translation operations, CAPTCHA-ROCK has a pass rate of $0.020 \%$ to attackers, even after the attacker has been given a careful tracing for each image in the control set.

- Is one trace per image enough?

Assume two histograms in Fig. 8 have an overlap. To assure the low pass rate for robots, we have to set a smaller threshold. In this case a false negative occurs: some legitimate attempts by humans would be denied. 
Our solution is to store multiple traces for one image. When a tentative solution is submitted by a user, we compare it to all traces and pass it if there is at least one distance below the threshold. Although human traces for the same image vary, by comparing to more than one "interpretation", the possibility of finding a close enough match increases. Note that this will not affect the pass rate for robot significantly. If a random trace is far from one trace, it is also far from other traces of the same image.

Based on our experiments which will be presented in Section 4.1, three traces per image improve the human pass rate, without helping the attackers.

- When can we promote an "unknown image" to a "control image"?

Once we have recorded three traces for an "unknown image", we promote it into the control set. Note that as a control image, the CAPTCHA-ROCK system will obtain more traces for the image. What should we do with these additional traces? We could ignore them, we could add them to the original three traces, or we could temporarily merge the new offering with the other three, expunging the one that has the furthest average distance from the others. We leave these considerations for future work.

\section{EXPERIMENTAL RESULTS}

\subsection{Quantitative Evaluation of CAPTCHA-ROCK System}

We have designed all experiments such that they are not only reproducible, but easily reproducible. To this end, we have built a webpage [17] which contains all datasets and code used in this work. In this section, we intend to show that:

- Our CAPTCHA-ROCK is very easy for humans to solve (high pass rate) and hard for robots (low pass rate).

- Storing multiple traces for each image helps increase the pass rate for humans, while not affecting the pass rate for robots significantly.

- A small "control image" set is sufficient, at least to bootstrap the system.

We randomly chose 143 images from our rock art image database, and had four volunteers draw traces for each image. The volunteers worked completely independely of each other. We call this initial trace dataset Trace_ini.

For reasons we will see shortly, we performed two rounds of rescaling and 2dimensional translation to each trace in Trace_ini. In the first round, we rescaled each trace image to $10 \% \sim 50 \%$ of its original size, and translated it in 2 dimensions by plus/minus $0 \sim 3$ times of the size in the $\mathrm{X}$ and $\mathrm{Y}$ axis independently. We call this new dataset Trace_robot. In the second round, every 4 traces of the same image in Trace_ini were performed by a same rescale and transition, and this dataset is called Trace_human.

As we assume that there is no automatic algorithm for extracting rock art data, we need to come up with an attack model. We make the pessimistic assumption that the attacker has our entire 143 image database, together with a human trace for each image. These seems to suggest that if the attacker simply submits a random tracing, he would have a one in 143 chance of passing the test, but recall that the images have been rescaled/translated in the plane. Such distortion means that even if the attacker happens to send the correct trace, it will probably not line up with the stored template, and will fail the test due to the penalty to the distance of centers of mass. 
We first tested the system with one randomly chosen (of four possibilities) trace from Trace_human for the "control image". To model the attacks from robots, each time we picked one trace from Trace_robot (but not those from the same person of the challenge trace). There were thus $3 \times 143$ tries for each challenge. As the human input, we picked the other three traces of the "control image" in Trace_human.

Using a threshold of seven, only 41 of 245,388 robot tests could pass, a pass rate of $0.014 \%$; whereas 1,632 of 1,716 human tests passed, a pass rate of $94.99 \%$.

Then we tested with three traces for each image. As noted in Section 3.2, each input from the user was compared to three traces of the "control image", and if its distance to one of them was below the threshold, the user passed the test. Each time we picked three traces of the same image from Trace_human as traces of the "control image". To model the attacks from robots, each time we picked one trace from the fourth person in Trace_robot. Thus, there were 143 tries for each challenge. As the human input we picked the remaining trace of the "control image" in Trace_human.

Using the same threshold of 7, only 22 of 81,796 robot tests could pass, a pass rate of $0.020 \%$; while only 5 of 572 human tests could not pass, a pass rate of $99.13 \%$.

Although our pass rate for robots is slightly larger than the generally accepted figure of $0.01 \%$ [6], note that all results are based on the initial control image set, with only 143 images. We expect the robot rate to decrease with more data, while the human rate should stay almost constant. Further recall that these results assume the pessimistic and unrealistic assumption that the attacker has traces for the entire database.

\subsection{Supporting Similarity Search}

The major goal of this work is to produce a dataset that will enable research by anthropologists. However, a minor goal is to produce a tool for non-specialists to query a database of petroglyphs. This tool could be used to support tourism [7], and to encourage an appreciation of indigenous people's cultural achievements.

We envision the following scenario: A hiker on a trail spots a petroglyph, and wants to know if it is known, and if so, what anthropologists and/or tribal historians have said about it. She photographs the petroglyph on her iPhone, traces the outline, and submits the query...

In order for this query to return the correct answer, our system must have several invariances. Some are trivial, as we are operating on a binary representation of the data, color and contrast invariance is automatically achieved ${ }^{1}$. However, as shown in Fig. 10, there need to be at least somewhat invariant in size, angle of view, etc.
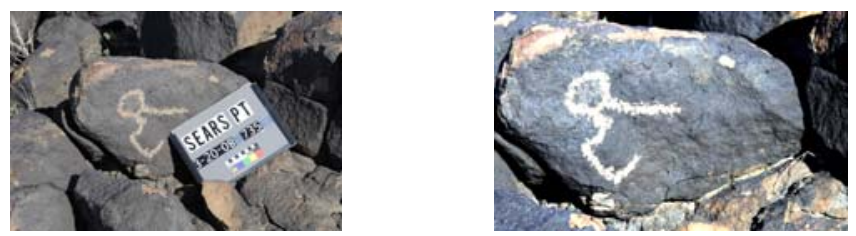

Fig. 10. Left) A petroglyph from Utah that has been indexed in our database. Right) An image of the same petroglyph found on Flickr.com. Could this image be used as a query to retrieve the anthropologist's annotated version in our database?

${ }^{1}$ For faint petroglyphs changing the contrast/color balance can enhance the petroglyphs visibility [9] [10] 
To test the feasibility of this scenario we obtained several examples of images of petroglyphs that we know are in our database (referred to the "control image" set in Section 4.1), but which were taken on a different day, by a different person, with a different camera etc. To normalize our expectations we also obtained photographs of petroglyphs that are known not to be in our database.

We had volunteers trace these petroglyph images. Note that in each case, these volunteers had not seen the data in the database, and were not familiar with our project.

Recall from the previous section that each petroglyph in our data collection had been traced by four independent volunteers. This means that for each petroglyph we had four models we could use to index it. We could also choose to have only one model for each petroglyph instead, by either averaging all four, or choosing the most typical one. However, here we kept all four models, both for simplicity, and because (as we shall see) it is instructive.

Our small dataset in this preliminary experiment does not warrant calculating precision/recall or similar statistics. Instead, we show typical results, and archive all results at [17]. Fig. 11 shows an example of a query using a (different photograph of) petroglyph that is in our database. The results are quite promising. Note that the query was taken from an image that was not as tightly cropped, and the user issuing the query (rightly or wrongly) traced a hook-like appendage on the left leg of the figure. Furthermore, note that among the four tracings in the database there is significant disagreement. For example, one individual did not trace the head as a circle. In spite of this, query-by-content is clearly successful in this example, as the first four matches are correct (the maximum possible).

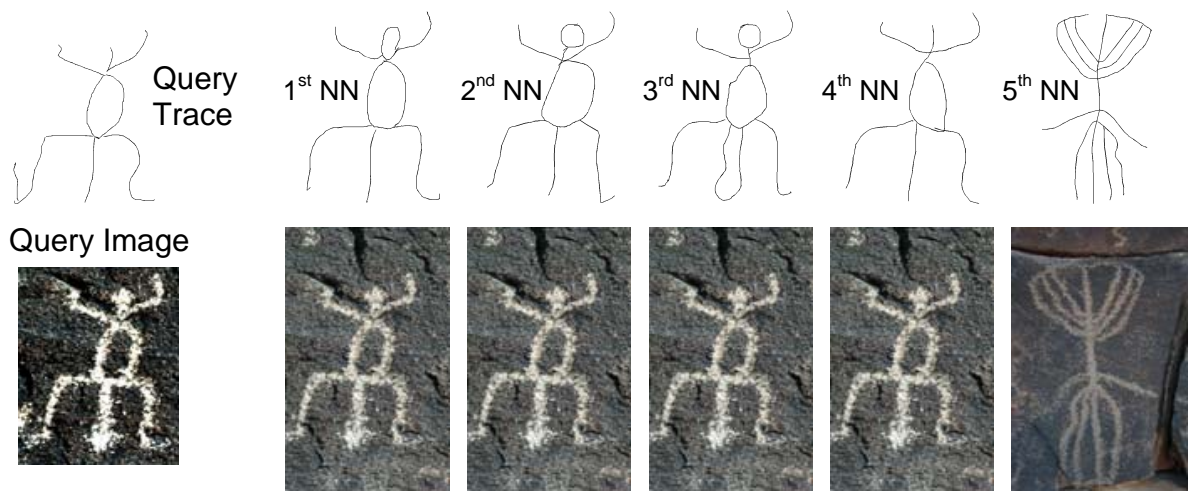

Fig. 11. left) A query petroglyph that happens to be in our database and its tracing. right) The five nearest neighbors to the query; the first four all refer to the same image, the correct target.

In Fig. 12, we see two more queries for which the relevant petroglyph (traced from a different photograph) is known to be in the database. For the "wheel" the $1^{\text {st }}, 2^{\text {nd }}$ and $4^{\text {th }}$ matches are correct, and the two others are at least plausible. For the bighorn sheep petroglyph, the first four matches are correct (the maximum possible), and the $5^{\text {th }}$ match is also plausible. 

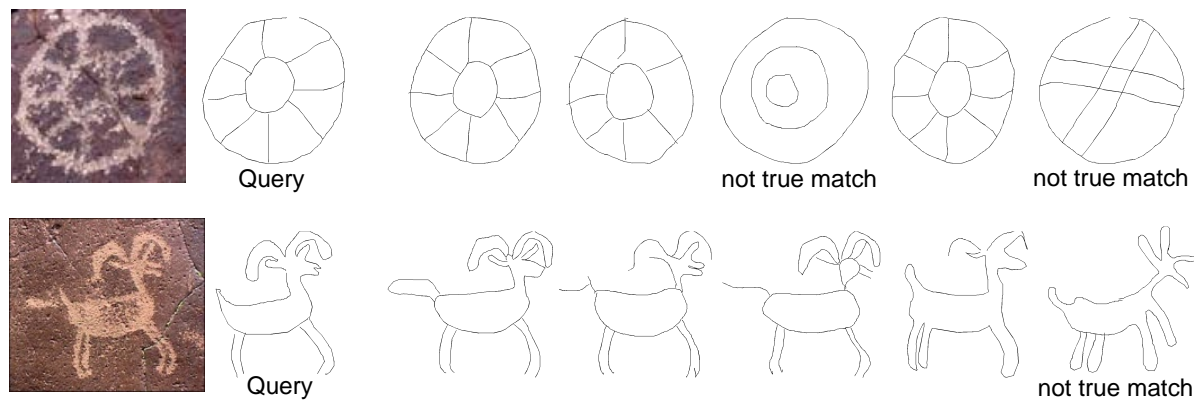
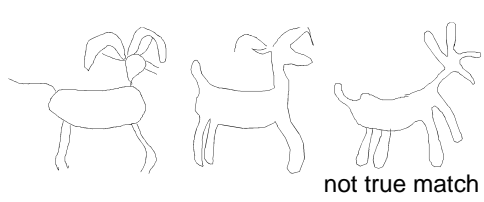

Fig. 12. An abstract (top) and animal petroglyph (bottom) which had been traced and issued as queries to our database. The list of the five nearest neighbors to each are shown left to right.

Finally, we consider the more difficult case, queries for which we know the relevant petroglyph is not in the database. Here the judgment of quality is subjective. Note also that we might expect to do better and better at this case as the database grows larger and larger. In Fig. 13 we show two queries and their best matches. In both cases the returned answers are reasonable.
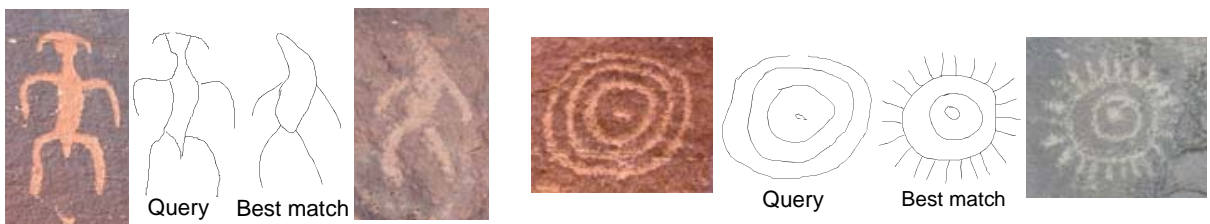

Fig. 13. left) An anthromorph is used as a query, and it retrieves another stylized human figure with similar limbs right) A petroglyph of concentric circles retrieves a "sunburst".

\section{CONCLUSIONS AND FUTURE WORK}

In this work, we consider the problem of digitizing large collections of rock art to enable the data mining of this neglected cultural resource. We have framed the feature extraction problem as a CAPTCHA, the first CAPTCHA we are aware of to produce and expect real-valued data. There are many private individuals that have on the order of hundreds of thousand of rock art images (i.e. the collection of Mark and Billo [10]), and Flicker.com appears to have on the order of one million rock art images. We believe that we could process all this data in less than one year, under realistic assumptions.

We note several limitations of our work. We could become victims of our own success, given that there are only tens of millions of rock images in existence, but there is a need for tens of millions of CAPTCHAs per day. So even if we capture only a small fraction of the CAPTCHA market, we may run out of rock art images. We believe that we may be able to bypass this issue by generating synthetic rock art images, in a spirit similar to the stickmen shown in Section 3.1. In addition, a significant fraction of petroglyphs images may not be amenable to our system; our CAPTCHA is not usable by blind users; and our system may be difficult to use on small screens such as iPhones. All these issues are the subject of ongoing research. 
Acknowledgements: This work was funded by NSF 0803410 and NSF 0808770. Field work for this project was funded by a National Geographic Society/Waitt Grant. We would like to thank the many donors of datasets, particularly Dr. Robert Mark and Evelyn Billo of www.rupestrian.com.

\section{REFERENCES}

[1] von Ahn,L., Blum,M., Hopper,N. and Langford, J. CAPTCHA: Using Hard AI Problems for Security. Advances in Cryptology, Lecture Notes in Computer Science, pp. 294-311.

[2] von Ahn, L., Maurer, B., McMillen, C., Abraham, D., and Blum, M. reCAPTCHA: Human-Based Character Recognition via Web Security Measures. Science. pp. 1465-1468. (2008) [3] von Ahn, L. Games with a purpose. Computer, 39(6):92-94. (2006)

[4] Aseyev, I. V. HORSEMAN IMAGE ON AN OSTRICH EGGSHELL FRAGMENT. Archaeology Ethnology \& Anthropology of Eurasia 34/2, 96-99. (2008)

[5] Ballard, D. H. Generalizing the Hough Transform to Detect Arbitrary Shapes, Pattern Recognition 13, 111-122. (1981)

[6] Chellapilla,K., Larson,K., Simard,P. and Czerwinski,M. Designing Human Friendly Human Interaction Proofs (HIPs). In Proceedings of ACM CHI 2005. pages 711-720.

[7] Dickinson, E. A. (under review). Evoking the Sacred: Commercial Appropriations of Nature in "The Petroglyphs." Western Journal of Communication.

[8] Henshilwood, CS et, al,. Emergence of Modern Human Behavior: Middle Stone Age Engravings from South Africa. Science 295:1278-1280. (2002)

[9] Landon,GV. and Seales.WB. Petroglyph Digitization: Enabling Cultural Heritage Scholarship, Machine Vision and Applications, vol. 17, no. 6, pp. 361-371, December (2006)

[10] Mark, R. K., and Billo, E. Application of Digital Image Enhancement in Rock Art Recording, American Indian Rock Art V. 28, 121-128. (2002)

[11] McDonald, J. J., Veth, P. M. Pilbara and Western Desert Rock Art: Style Graphics in

Arid Landscapes. Proceedings of the XXII Valcamonica Symposium. pp. 327-334. (2007)

[12] Merlin, P. M., and Farber, D. J. A Parallel Mechanism for Detecting Curves in Pictures, IEEE Trans. Comput. C24, 96-98 (1975).

[13] Pettigrew, J., Nugent, M., McPhee, A.,Wallman, J. An Unexpected, Stripe-faced Flying Fox in Ice Age Rock Art of Australia's Kimberley. Journal of Antiquity. (2008)

[14] Valladas,H., Clottes,J., Geneste,J-M., Garcia,MA., Arnold,M., Cachier,H. and TisnératLaborde,N. Palaeolithic Paintings: Evolution of Prehistoric Cave Art. Nature 413:479. (2001)

[15] Walt, H., David, B., Brayer, J. \& Musello, C. The Rock Art Database Project.

[16] Zhu, Q., Wang, X., Keogh, E. and Lee, SH. Augmenting the Generalized Hough Transform to Enable the Mining of Petroglyphs. KDD 2009: 1057-1066 (2009)

[17] Zhu, Q. CAPTCHA Rock Webpage:

http://www.cs.ucr.edu/ qzhu/CAPTCHA_Rock.html (2010) 